J. Product. \& Dev., 23(3): 535- 554(2018)

\title{
EFFECT OF SOME NANO PARTICLE MATERIALS ON LEAF AREA, PHOTO SYNTHETIC PIGMENTS AND MINERALS CONTENT OF MANGO TREES
}

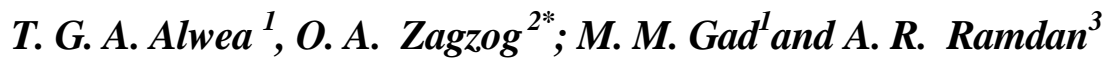 \\ 1 Hort. Depart. Fac. of Agric., Zagazig Univ., Egypt. mmgad@zu.edu.eg \\ 2 Plant Prod. Depart. Fac. of Tech. and Deve., Zagazig Univ., Egypt. \\ 3 Rese. of Techno. Hort. Crops Depart, National Rese. Center, Egypt. \\ *zagzog_1000@yahoo.com
}

\section{ABSTRACT}

This study was carried out during the two growing consecutive seasons 2015 / 2016 on mango trees (Mangifera indica L.) cvs. Zebda and Ewasy, grown in a private orchard at Wadi El-Muolak, Sharkia Governorate, Egypt to evaluate the effect of foliar chitosan and potassium silicate components nano or not nano particle at 15 February before flowering on average leaf area and leaf content of pigments and minerals. Ewasy $\mathrm{cv}$. displayed the highest of their tested attributes in most cases A, $B$, total Chlorophyll and leaf mineral content compared with Zebda cv.

The obtained showed that the tested treatments improved leaf characteristics especially nano chitosan 50 and 150 ppm treatments which increased Chlorophyll B, total Chlorophyll as well as leaf mineral content in leaf. Furthermore, the trees were sprayed with nano-silica 0.6 and 0.8 $\mathrm{g} / \mathrm{L}$ showed the highest average leaf area Chlorophyll A and caroteniods pigments in leaf.

Conclusively, from results of this research, the resembling conditions that spraying mango trees nano-nano chitosan 50 and $150 \mathrm{ppm}$ nano-silica 0.6 and $0.8 \mathrm{~g} / \mathrm{L}$ before flowering is desirable for improving leaf characteristics it is recommending, under Belbeis district, Sharkia Governorate, Egypt

Key words: Mango, nano chitosan, nano silicate, Chlorophyll, leaf mineral, Zebda, Ewasy. 


\section{INTRODUCTION}

Mango (Mangifera indica L.) is one of the most important tropical fruits in the world (Mukherjee, 1951) and one of the most popular fruits in Egypt. The total area of the Egyptian mangoes reached 281153 feds. (Statistics of the Ministry of Agriculture, 2016).

Chitosan is considered a biopolymer produced from chitin and is very safe for human being. It has bioactivity and bio-compatibility (Dias et al., 2013). Using it in plants resulted in improving the yield and reducing transpiration (Dzung et al., 2011 and Mondal et al., 2012). It is responsible for enhancing the tolerance of plants to bacterial, viral and fungal attack (Al-Hetar et al., 2011). The plants subjected to chitosan are less prone to all biotic and abiotic stresses (Lizarraga-Paulin et al., 2011, Jabeen and Ahmad, 2013 and Pongprayoon et al., 2013). Previous studies emphasized the essential role of chitosan on stimulating growth aspects, as well as, improving yield and quality parameters of fruits (Chibu and Shibayama, 1999, Li and Yu, 2000, Bittelli et al., 2001 and Devlieghere et al., 2004 and Hussein Esraa, 2017).

Silicon has many functions in plant nutrition. It has many regulatory roles in enhancing the tolerance of plants to biotic and abiotic stresses, water retention, photosynthesis, plant pigments and building of carbohydrates and natural growth regulators (Gang et al., 2003, Lux et al., 2003, Ma, 2004 and Hattori et al., 2005). Previous studies (Gad El-Kareem et al., 2014, Omar, 2015, Ahmed et al., 2017 and Youssef, 2017) showed that silicon were favorable in enhancing vegetative growth characteristics and leaf mineral content in various fruit trees.

Therefore, the main objective of this study is to investigate the effect of nano particle of chitosan and potassium silicate on leaf area, pigments and minerals contents of two mangoes cultivars Zebda and Ewasy.

\section{MATERIALS AND METHODS}

This investigation was carried out on two mangoes (Mangifera indica L.) cultivars Zebda and Ewasy in 2016 and 2017 seasons on mango trees grown in sandy soil under drip irrigation at private orchard at Wadi El-Muluk, Sharkia Governorate, Egypt.

Trees were 20 years old, planted at $4 \times 6 \mathrm{~m}$ apart, grafted on Succary rootstocks grown under the common agricultural practices adopted in the area. Seventy eight healthy trees, similar in vigor and size, selected to evaluate the 
effect of foliar spray with different of treatments before flowering at 15 February on leaf area and leaf content of pigments and minerals of mango cultivars Ewasy and Zebda.

Chitosan and potassium silicate of nano crystallite powder synthesized by high-energy ball milling. Powder mixture conducted in a 0.4 mini lab planetary ball mill (model DECO-PBM-V-0.4L, Changsha Deco Equipment Co., Ltd., China) to $40 \mathrm{~h}$ using ball to powder mass ratio of 8:1 by prof. Dr. Osama M. Hemeda at Central lab., department of physics, faculty of science, Tanta University, Egypt. The microstructure of the sintered samples examined using High Resolution Transmission Electron Microscope (HRTEM) model JOEL EM 2-100. Transmission electron microscope (TEM) imaging showed a spherical, smooth and almost homogenous structure for nanoparticles. In the present study, TEM images (Fig. 1) have shown the morphological properties and surface appearance of chitosan nanoparticles, which have nearly spherical shape, smooth surface and size range of about $50-35 \mathrm{~nm}$, which confirm the result of XRD. The size of chitosan nanoparticles, as evident from the TEM images found to be $50 \mathrm{~nm}$. The TEM analysis of chitosan nanoparticles showed uniform size distribution in nanometer range.

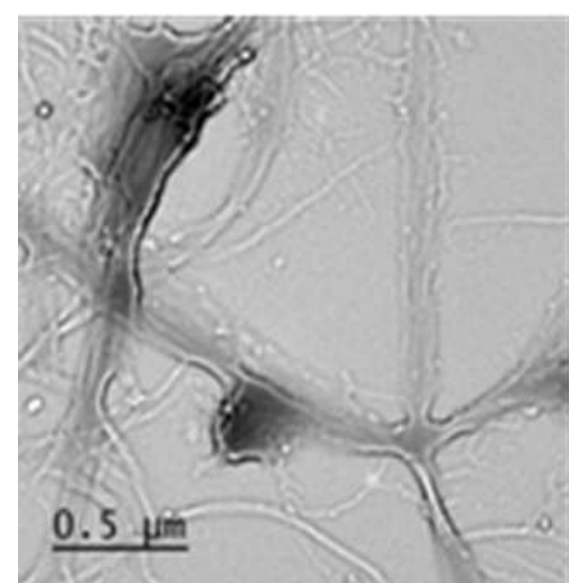

(a)

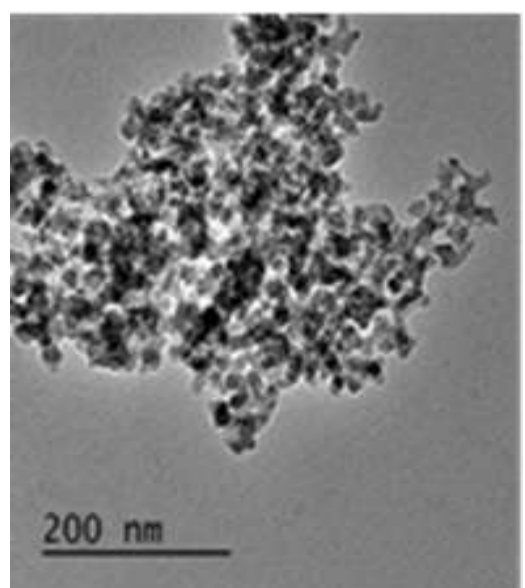

(b)

Fig.1 (a-b): TEM micrograph for chitosan, (a) before milling and (b) after milling 


\section{Chitosan dissolving:}

For experimental use the stock solution, $(2.0 \%$, w/v) of chitosan was prepared by dissolving purified chitosan in $0.5 \%(\mathrm{v} / \mathrm{v})$ glacial acetic acid (Du et al., 1997), under continuous stirring, and the $\mathrm{pH}$ was adjusted to 5.6 using $1 \mathrm{~N}$ $\mathrm{Na} \mathrm{OH}$. The stock solution was autoclaved and appropriate concentrations were obtained by dilution with sterile distilled water.

Thirteen treatments each treatment included 3 replicates. The replicate represented as one tree of every cultivar were applied on the two cultivars as follows:

1- Trees sprayed only mineral oil $1.5 \%$ (control).

2- Trees sprayed with nano chitosan $50 \mathrm{ppm}$ ( $2.5 \mathrm{ml} / 1$ from stock solution) + mineral oil $1 \%$.

3- Trees sprayed with nano chitosan $100 \mathrm{ppm}(5 \mathrm{ml} / \mathrm{l}$ from stock solution) + mineral oil $1.5 \%$.

4- Trees sprayed with nano chitosan $150 \mathrm{ppm}$ (7.5 ml/l from stock solution) + mineral oil $1.5 \%$.

5- Trees sprayed with chitosan $50 \mathrm{ppm}+$ mineral oil $1 \%$.

6- Trees sprayed with chitosan $100 \mathrm{ppm}+$ mineral oil $1.5 \%$.

7- Trees sprayed with chitosan $150 \mathrm{ppm}+$ mineral oil $1.5 \%$.

8- Trees sprayed with nano potassium silicate $0.4 \mathrm{~g} / \mathrm{l}(40 \mathrm{~g} / 100 \mathrm{~L})$.

9- Trees sprayed with nano potassium silicate $0.6 \mathrm{~g} / \mathrm{l}(60 \mathrm{~g} / 100 \mathrm{~L})$.

10- Trees sprayed with nano potassium silicate $0.8 \mathrm{~g} / \mathrm{l}(80 \mathrm{~g} / 100 \mathrm{~L})$.

11- Trees sprayed with potassium silicate $0.4 \mathrm{~g} / \mathrm{l}(40 \mathrm{~g} / 100 \mathrm{~L})$.

12- Trees sprayed with potassium silicate $0.6 \mathrm{~g} / \mathrm{l}(60 \mathrm{~g} / 100 \mathrm{~L})$.

13- Trees sprayed with potassium silicate $0.8 \mathrm{~g} / \mathrm{l}(80 \mathrm{~g} / 100 \mathrm{~L})$.

The responses of the two mango cvs. to the applied treatments were evaluated through the following parameters:

\section{Leaf characteristics:}

Average leaf area:

To determine average leaf area (indicators to plant growth effects), samples of mature leaves grown on unfruitful shoots randomly taken at harvest date length and width of leaf $(\mathrm{cm})$ were measured. Then, average leaf surface area $\left(\mathrm{cm}^{2}\right)$ was determined according to the equation reported by Ahmed and Morsy (1999).

Leaf area $\left(\mathrm{cm}^{2}\right)=0.7$ (blade width $\mathrm{x}$ blade length) -1.06 . 


\section{Leaf pigments contents:}

Leaf disk samples obtained at harvest to determined leaf tissue contents (mg / mg F.W) of chlorophyll A, B, total chlorophyll $(\mathrm{A}+\mathrm{B})$ and carotenoids (Wettestein, 1957).

\section{Leaf mineral contents:}

Leaf samples were washed, dried ground and digested using sulphoric acid and hydrogen peroxide (Chapman and Pratt 1961). N, P, K and Zn content were determined in the digested solution as percentages. All chemicals used in different determinations obtained from El-Gomhouria for trading Chemicals and Medical Appliances, El-Sawah, El-Amiria, Cairo, Egypt.

\section{Statistical analysis:}

This study designed as factorial experimental with to factors; cultivars (2) and treatments (13). data were analyzed using M-Stat program in a randomized complete block design (RCBD) as described by Snedecor and Cochran (1980), and means were compared using mean comparison at 0.05 level (Duncan, 1955).

\section{RESULTS}

\section{Average leaf area:}

Data in Table 1 revealed that the average leaf area of Ewasy was significantly affected and largest compared to Zebda cv. in both seasons.

The tested treatments showed significant effects on average leaf area. The Nano-silica treatments recorded the highest values compared of other treatments. Whilst, trees sprayed with nano-silica 0.6 and $0.8 \mathrm{~g} / \mathrm{L}$ showed the highest average leaf area $\left(140.46 \& 128.73\right.$ and $\left.142.28 \& 145.01 \mathrm{~cm}^{2}\right)$ in the first and second seasons, respectively without significant differences between them. While, the lowest average leaf area $\left(43.95 \& 51.09\right.$ and $\left.59.43 \& 51.79 \mathrm{~cm}^{2}\right)$ in the first and second seasons, respectively without significant differences between them was recorded for trees sprayed with control and chitosan $50 \mathrm{ppm}$ treatments.

The interaction between Ewasy $\times$ nano-silica 0.6 and $0.8 \mathrm{~g} / \mathrm{L}$ had superior values of average leaf area, compared with Zebda $\times$ control and chitosan treatments which were in significant differences between them in both seasons. 
Table (1): Effect of chitosan and potassium silicate spraying on average leaf area

$\left(\mathrm{cm}^{2}\right)$ of Zebda and Ewasy mango trees (2016 and 2017 seasons)

\begin{tabular}{|c|c|c|c|c|c|c|}
\hline \multirow{3}{*}{ Treatments } & \multicolumn{3}{|c|}{ First season (2016) } & \multicolumn{3}{|c|}{ Second season (2017) } \\
\hline & \multicolumn{2}{|c|}{ Cultivar } & \multirow{2}{*}{ Treat. av. } & \multicolumn{2}{|c|}{ Cultivar } & \multirow{2}{*}{ Treat. av. } \\
\hline & Zebda & Ewasy & & Zebda & Ewasy & \\
\hline Control (water) & 27.761 & $37.44 \mathrm{kl}$ & $43.95 \mathrm{E}$ & $36.62 \mathrm{jk}$ & 82.24 efg & $59.43 \mathrm{E}$ \\
\hline Chitosan 50 ppm & $60.14 \mathrm{ij}$ & 64.74 ghij & $51.09 \mathrm{E}$ & $39.31 \mathrm{jk}$ & $64.27 \mathrm{hi}$ & $51.79 \mathrm{E}$ \\
\hline Chitosan 100 ppm & $52.09 \mathrm{ijk}$ & 95.19 ef & 73.64 D & $48.52 \mathrm{ijk}$ & $95.77 \mathrm{de}$ & $72.15 \mathrm{D}$ \\
\hline Chitosan 150 ppm & $39.42 \mathrm{kl}$ & 67.54 ghi & $53.48 \mathrm{E}$ & $31.02 \mathrm{k}$ & 84.69 ef & $57.86 \mathrm{E}$ \\
\hline Nano-chitosan 50 ppm & $51.79 \mathrm{ijk}$ & 90.29 ef & $71.04 \mathrm{D}$ & $63.08 \mathrm{hi}$ & $92.51 \mathrm{de}$ & $77.80 \mathrm{D}$ \\
\hline Nano-chitosan 100 ppm & $61.24 \mathrm{ij}$ & $79.98 \mathrm{fgh}$ & $70.61 \mathrm{D}$ & $57.48 \mathrm{hi}$ & $99.13 \mathrm{de}$ & $78.31 \mathrm{D}$ \\
\hline Nano-chitosan 150 ppm & $49.46 \mathrm{jk}$ & 87.96 ef & $68.71 \mathrm{D}$ & 53.89 hij & $103.94 \mathrm{~d}$ & $78.91 \mathrm{D}$ \\
\hline Silica $0.4 \mathrm{~g} / \mathrm{L}$ & $56.81 \mathrm{ij}$ & $148.39 \mathrm{c}$ & $102.60 \mathrm{C}$ & 65.32 ghi & $161.11 \mathrm{c}$ & 113.22 C \\
\hline Silica $0.6 \mathrm{~g} / \mathrm{L}$ & $82.36 \mathrm{fg}$ & $185.37 \mathrm{~b}$ & 133.87 AB & $70.20 \mathrm{fgh}$ & $195.17 \mathrm{~b}$ & $132.69 \mathrm{AB}$ \\
\hline Silica $0.8 \mathrm{~g} / \mathrm{L}$ & $101.14 \mathrm{e}$ & $152.92 \mathrm{c}$ & & $89.94 \mathrm{de}$ & $160.41 \mathrm{c}$ & 125.17 BC \\
\hline Nano-silica $0.4 \mathrm{~g} / \mathrm{L}$ & $57.86 \mathrm{ij}$ & $131.36 \mathrm{~d}$ & $94.61 \mathrm{C}$ & $63.22 \mathrm{hi}$ & $170.67 \mathrm{c}$ & $116.95 \mathrm{C}$ \\
\hline Nano-silica $0.6 \mathrm{~g} / \mathrm{L}$ & $78.27 \mathrm{fgh}$ & $202.64 \mathrm{a}$ & $140.46 \mathrm{~A}$ & 65.95 ghi & $218.60 \mathrm{a}$ & $142.28 \mathrm{~A}$ \\
\hline Nano-silica $0.8 \mathrm{~g} / \mathrm{L}$ & 64.27 hij & $193.19 \mathrm{ab}$ & $128.73 \mathrm{AB}$ & 65.67 ghi & $224.34 \mathrm{a}$ & 145.01 A \\
\hline Cultivar av. & $58.45 \mathrm{~B}$ & $119.98 \mathrm{~A}$ & & $57.71 \mathrm{~B}$ & 134.84 A & \\
\hline
\end{tabular}

Means having the same letter (s) within the same column are not significantly different according to Duncan's multiple range test at $5 \%$ level of probability.

\section{Leaf photosynthetic pigments:}

Data in Tables 2 - 5 showed that leaf content of Chlorophyll B and total was significantly differed between the two tested mango varieties but, leaf Chlorophyll was total all insignificant in the leaves in the second season only. While, leaf content of carotenoids was insignificantly between two cultivars.

The data cleared also that leaf photosynthetic pigments were significantly response to treating the trees. The treatments with silica at $0.6 \mathrm{~g} / \mathrm{L}$ gave the highest values of leaf Chlorophyll A and carotene content $(1.697 \& 1.743$ and $2.789 \& 2.953 \mathrm{mg} / 100 \mathrm{mg}$ ) in the first and second seasons, respectively. Treating the trees with nano chitosan $50 \mathrm{ppm}$ gave the highest values of leaf 
Table (2): Effect of chitosan and potassium silicate spraying on chlorophyll A content (mg/ $100 \mathrm{mg}$ F.W.) of Zebda and Ewasy mango leaves (2016 and 2017 seasons)

\begin{tabular}{|c|c|c|c|c|c|c|}
\hline \multirow{3}{*}{ Treatments } & \multicolumn{3}{|c|}{ First season (2016) } & \multicolumn{3}{|c|}{ Second season (2017) } \\
\hline & \multicolumn{2}{|c|}{ Cultivar } & \multirow{2}{*}{ Treat. av. } & \multicolumn{2}{|c|}{ Cultivar } & \multirow{2}{*}{ Treat. av. } \\
\hline & Zebda & Ewasy & & Zebda & Ewasy & \\
\hline Control (water) & $0.818 \mathrm{p}$ & 0.909 o & $0.864 \mathrm{~L}$ & $0.931 \mathrm{~m}$ & $0.894 \mathrm{~m}$ & 0.913 I \\
\hline Chitosan 50 ppm & $1.135 \mathrm{~m}$ & 1.2241 & $1.180 \mathrm{~J}$ & 1.1151 & $1.217 \mathrm{jk}$ & $1.166 \mathrm{G}$ \\
\hline Chitosan 100 ppm & $1.317 \mathrm{j}$ & $1.515 \mathrm{f}$ & $1.416 \mathrm{~F}$ & $1.420 \mathrm{fg}$ & $1.320 \mathrm{i}$ & $1.370 \mathrm{D}$ \\
\hline Chitosan 150 ppm & 1.2101 & $1.304 \mathrm{j}$ & $1.257 \mathrm{H}$ & $1.233 \mathrm{jk}$ & $1.495 \mathrm{e}$ & $1.364 \mathrm{D}$ \\
\hline Nano-chitosan 50 ppm & $1.448 \mathrm{~g}$ & $1.682 \mathrm{~d}$ & $1.565 \mathrm{C}$ & $1.653 \mathrm{c}$ & $1.554 \mathrm{~d}$ & $1.604 \mathrm{~B}$ \\
\hline Nano-chitosan 100 ppm & 1.2051 & $1.885 \mathrm{~b}$ & $1.545 \mathrm{D}$ & $1.318 \mathrm{i}$ & $1.380 \mathrm{gh}$ & $1.349 \mathrm{D}$ \\
\hline Nano-chitosan 150 ppm & 1.2121 & $1.424 \mathrm{~h}$ & $1.318 \mathrm{G}$ & 1.1121 & $1.612 \mathrm{c}$ & $1.362 \mathrm{D}$ \\
\hline Silica $0.4 \mathrm{~g} / \mathrm{L}$ & $1.045 \mathrm{n}$ & $1.045 \mathrm{n}$ & $1.045 \mathrm{~K}$ & $2.305 \mathrm{a}$ & $1.222 \mathrm{jk}$ & $1.764 \mathrm{~A}$ \\
\hline Silica $0.6 \mathrm{~g} / \mathrm{L}$ & $1.651 \mathrm{e}$ & $1.743 \mathrm{c}$ & $1.697 \mathrm{~A}$ & $1.854 \mathrm{~b}$ & $1.632 \mathrm{c}$ & $1.743 \mathrm{~A}$ \\
\hline Silica $0.8 \mathrm{~g} / \mathrm{L}$ & $1.142 \mathrm{~m}$ & 1.2131 & $1.178 \mathrm{~J}$ & 1.0981 & 1.1241 & $1.111 \mathrm{H}$ \\
\hline Nano-silica $0.4 \mathrm{~g} / \mathrm{L}$ & 1.2051 & $1.260 \mathrm{k}$ & $1.232 \mathrm{I}$ & $1.197 \mathrm{k}$ & $1.248 \mathrm{j}$ & $1.222 \mathrm{~F}$ \\
\hline Nano-silica $0.6 \mathrm{~g} / \mathrm{L}$ & $1.400 \mathrm{i}$ & $1.515 \mathrm{f}$ & $1.457 \mathrm{E}$ & $1.505 \mathrm{e}$ & $1.425 \mathrm{f}$ & $1.465 \mathrm{C}$ \\
\hline Nano-silica $0.8 \mathrm{~g} / \mathrm{L}$ & $1.697 \mathrm{a}$ & $1.306 \mathrm{j}$ & $1.650 \mathrm{~B}$ & $1.255 \mathrm{j}$ & $1.359 \mathrm{hi}$ & $1.307 \mathrm{E}$ \\
\hline Cultivar av. & $1.291 \mathrm{~B}$ & $1.387 \mathrm{~A}$ & & $1.384 \mathrm{~A}$ & $1.345 \mathrm{~B}$ & \\
\hline
\end{tabular}

Means having the same letter (s) within the same column are not significantly different according to Duncan's multiple range test at $5 \%$ level of probability. 
Table (3): Effect of chitosan and potassium silicate spraying on chlorophyll B content (mg/ $100 \mathrm{mg}$ F.W.) of Zebda and Ewasy mango leaves (2016 and 2017 seasons)

\begin{tabular}{|c|c|c|c|c|c|c|}
\hline \multirow{3}{*}{ Treatments } & \multicolumn{3}{|c|}{ First season (2016) } & \multicolumn{3}{|c|}{ Second season (2017) } \\
\hline & \multicolumn{2}{|c|}{ Cultivar } & \multirow{2}{*}{ Treat. av. } & \multicolumn{2}{|c|}{ Cultivar } & \multirow{2}{*}{ Treat. av. } \\
\hline & Zebda & Ewasy & & Zebda & Ewasy & \\
\hline Control (water) & $1.216 \mathrm{w}$ & $1.311 \mathrm{v}$ & $1.263 \mathrm{~L}$ & $1.310 \mathrm{kl}$ & 1.2361 & $1.273 \mathrm{~F}$ \\
\hline Chitosan 50 ppm & $1.521 \mathrm{r}$ & $1.628 \mathrm{n}$ & $1.574 \mathrm{~J}$ & 1.563 ghi & $1.661 \mathrm{e}-\mathrm{i}$ & $1.612 \mathrm{D}$ \\
\hline Chitosan 100 ppm & $1.447 \mathrm{~s}$ & $1.836 \mathrm{~h}$ & $1.641 \mathrm{H}$ & $1.663 \mathrm{~d}-\mathrm{i}$ & $1.725 \mathrm{~d}-\mathrm{g}$ & 1.694 CD \\
\hline Chitosan 150 ppm & $1.624 \mathrm{o}$ & 1.7041 & $1.664 \mathrm{G}$ & $1.588 \mathrm{f}-\mathrm{i}$ & $1.716 \mathrm{~d}-\mathrm{g}$ & $1.652 \mathrm{CD}$ \\
\hline Nano-chitosan 50 ppm & $2.182 \mathrm{a}$ & $1.865 \mathrm{~g}$ & $2.024 \mathrm{~A}$ & $2.217 \mathrm{a}$ & $1.924 \mathrm{bc}$ & $2.071 \mathrm{~A}$ \\
\hline Nano-chitosan 100 ppm & $1.634 \mathrm{~m}$ & $1.992 \mathrm{~b}$ & $1.813 \mathrm{C}$ & $1.713 \mathrm{~d}-\mathrm{g}$ & 1.784 cde & 1.479 BC \\
\hline Nano-chitosan 150 ppm & $1.423 \mathrm{t}$ & $1.953 \mathrm{c}$ & $1.688 \mathrm{~F}$ & $1.563 \mathrm{ghi}$ & 1.803 cde & $1.683 \mathrm{CD}$ \\
\hline Silica $0.4 \mathrm{~g} / \mathrm{L}$ & $1.334 \mathrm{u}$ & $1.562 \mathrm{p}$ & $1.458 \mathrm{~K}$ & $1.505 \mathrm{hij}$ & $1.347 \mathrm{jkl}$ & $1.426 \mathrm{E}$ \\
\hline Silica $0.6 \mathrm{~g} / \mathrm{L}$ & $1.217 \mathrm{w}$ & $1.944 \mathrm{~d}$ & 1.580 I & $0.736 \mathrm{n}$ & $1.763 \mathrm{c}-\mathrm{f}$ & $1.250 \mathrm{~F}$ \\
\hline Silica $0.8 \mathrm{~g} / \mathrm{L}$ & $1.876 \mathrm{f}$ & $1.547 \mathrm{q}$ & $1.711 \mathrm{E}$ & $2.042 \mathrm{ab}$ & $1.472 \mathrm{ijk}$ & 1.757 BC \\
\hline Nano-silica $0.4 \mathrm{~g} / \mathrm{L}$ & $1.621 \mathrm{o}$ & $1.708 \mathrm{k}$ & $1.664 \mathrm{G}$ & $1.538 \mathrm{~g}-\mathrm{j}$ & $1.695 \mathrm{~d}-\mathrm{h}$ & $1.616 \mathrm{D}$ \\
\hline Nano-silica $0.6 \mathrm{~g} / \mathrm{L}$ & $1.764 \mathrm{j}$ & $1.923 \mathrm{e}$ & $1.843 \mathrm{~B}$ & $1.805 \mathrm{cde}$ & $1.840 \mathrm{~cd}$ & $1.823 \mathrm{~B}$ \\
\hline Nano-silica $0.8 \mathrm{~g} / \mathrm{L}$ & $1.634 \mathrm{~m}$ & $1.794 \mathrm{i}$ & $1.714 \mathrm{D}$ & $1.031 \mathrm{~m}$ & $1.713 \mathrm{~d}-\mathrm{g}$ & $1.372 \mathrm{EF}$ \\
\hline Cultivar av. & $1.576 \mathrm{~B}$ & $1.751 \mathrm{~A}$ & & $1.560 \mathrm{~B}$ & $1.668 \mathrm{~A}$ & \\
\hline
\end{tabular}

Means having the same letter (s) within the same column are not significantly different according to Duncan's multiple range test at $5 \%$ level of probability. 
Table (4): Effect of chitosan and potassium silicate spraying on total chlorophyll content (mg/ $100 \mathrm{mg}$ F.W.) of Zebda and Ewasy mango leaves (2016 and 2017 seasons)

\begin{tabular}{|c|c|c|c|c|c|c|}
\hline \multirow{3}{*}{ Treatments } & \multicolumn{3}{|c|}{ First season (2016) } & \multicolumn{3}{|c|}{ Second season (2017) } \\
\hline & \multicolumn{2}{|c|}{ Cultivar } & \multirow{2}{*}{ Treat. av. } & \multicolumn{2}{|c|}{ Cultivar } & \multirow{2}{*}{ Treat. av } \\
\hline & Zebda & Ewasy & & Zebda & Ewasy & \\
\hline Control (water) & $2.034 \mathrm{u}$ & $2.220 \mathrm{t}$ & $2.127 \mathrm{~K}$ & $2.241 \mathrm{~m}$ & $2.130 \mathrm{~m}$ & $2.186 \mathrm{G}$ \\
\hline Chitosan 50 ppm & $2.655 \mathrm{p}$ & $2.852 \mathrm{~m}$ & $2.753 \mathrm{I}$ & $2.291 \mathrm{~lm}$ & 2.877 hij & $2.584 \mathrm{~F}$ \\
\hline Chitosan 100 ppm & $2.764 \mathrm{o}$ & $3.351 \mathrm{~g}$ & 3.057 E & $3.084 \mathrm{e}-\mathrm{h}$ & $3.045 \mathrm{e}-\mathrm{i}$ & 3.064 CD \\
\hline Chitosan 150 ppm & $2.834 \mathrm{mn}$ & $3.008 \mathrm{j}$ & $2.921 \mathrm{G}$ & $2.820 \mathrm{ijk}$ & $3.211 \mathrm{c}-\mathrm{f}$ & $3.016 \mathrm{D}$ \\
\hline Nano-chitosan 50 ppm & $3.630 \mathrm{c}$ & $3.547 \mathrm{~d}$ & $3.588 \mathrm{~A}$ & $3.789 \mathrm{a}$ & $3.478 \mathrm{~b}$ & $3.633 \mathrm{~A}$ \\
\hline Nano-chitosan 100 ppm & $2.839 \mathrm{mn}$ & $3.877 \mathrm{a}$ & $3.358 \mathrm{~B}$ & $3.031 \mathrm{f}-\mathrm{i}$ & $3.164 \mathrm{~d}-\mathrm{g}$ & 3.098 CD \\
\hline Nano-chitosan 150 ppm & $2.635 \mathrm{q}$ & $3.377 \mathrm{f}$ & $3.006 \mathrm{~F}$ & $2.674 \mathrm{jk}$ & $3.415 \mathrm{bc}$ & 3.045 CD \\
\hline Silica $0.4 \mathrm{~g} / \mathrm{L}$ & $2.378 \mathrm{~s}$ & $2.607 \mathrm{r}$ & $2.493 \mathrm{~J}$ & $3.810 \mathrm{a}$ & $2.569 \mathrm{kl}$ & 3.190 BC \\
\hline Silica $0.6 \mathrm{~g} / \mathrm{L}$ & 2.8721 & $3.687 \mathrm{~b}$ & $3.279 \mathrm{D}$ & $3.264 b-f$ & $3.395 \mathrm{bcd}$ & $3.329 \mathrm{~B}$ \\
\hline Silica $0.8 \mathrm{~g} / \mathrm{L}$ & $3.01 \mathrm{j}$ & $2.760 \mathrm{o}$ & $2.889 \mathrm{H}$ & $2.909 \mathrm{~g}-\mathrm{j}$ & $2.596 \mathrm{k}$ & 2.753 EF \\
\hline Nano-silica $0.4 \mathrm{~g} / \mathrm{L}$ & $2.826 n$ & $2.967 \mathrm{k}$ & $2.896 \mathrm{H}$ & $2.734 \mathrm{jk}$ & $2.942 \mathrm{~g}-\mathrm{j}$ & $2.838 \mathrm{E}$ \\
\hline Nano-silica $0.6 \mathrm{~g} / \mathrm{L}$ & $3.163 \mathrm{~h}$ & $3.439 \mathrm{e}$ & 3.301 C & $3.311 \mathrm{~b}-\mathrm{e}$ & $3.264 b-f$ & $3.2878 \mathrm{~B}$ \\
\hline Nano-silica $0.8 \mathrm{~g} / \mathrm{L}$ & $3.626 \mathrm{c}$ & $3.100 \mathrm{i}$ & $3.363 \mathrm{~B}$ & $2.287 \mathrm{~lm}$ & $3.073 \mathrm{e}-\mathrm{i}$ & 2.680 EF \\
\hline Cultivar av. & $2.867 \mathrm{~B}$ & $3.138 \mathrm{~A}$ & & $2.942 \mathrm{~A}$ & $3.012 \mathrm{~A}$ & \\
\hline
\end{tabular}

Means having the same letter (s) within the same column are not significantly different according to Duncan's multiple range test at $5 \%$ level of probability. 
Table (5): Effect of chitosan and potassium silicate spraying on carotenoids content (mg/ $100 \mathrm{mg}$ F.W.) of Zebda and Ewasy mango leaves (2016 and 2017 seasons)

\begin{tabular}{|c|c|c|c|c|c|c|}
\hline \multirow{3}{*}{ Treatments } & \multicolumn{3}{|c|}{ First season (2016) } & \multicolumn{3}{|c|}{ Second season (2017) } \\
\hline & \multicolumn{2}{|c|}{ Cultivar } & \multirow{2}{*}{ Treat. av. } & \multicolumn{2}{|c|}{ Cultivar } & \multirow{2}{*}{ Treat. av. } \\
\hline & Zebda & Ewasy & & Zebda & Ewas & \\
\hline Control (water) & $1.837 \mathrm{i}$ & $1.815 \mathrm{i}$ & $1.826 \mathrm{~F}$ & $1.915 \mathrm{~h}-\mathrm{k}$ & $1.741 \mathrm{jk}$ & $1.828 \mathrm{~F}$ \\
\hline Chitosan 50 ppm & $2.083 \mathrm{gh}$ & $2.221 \mathrm{efg}$ & $2.152 \mathrm{E}$ & $2.112 \mathrm{f}-\mathrm{j}$ & $2.156 \mathrm{e}-\mathrm{i}$ & 2.134 CDE \\
\hline Chitosan 100 ppm & $2.211 \mathrm{efg}$ & $2.479 \mathrm{bc}$ & $2.345 \mathrm{BC}$ & $2.287 \mathrm{c}-\mathrm{h}$ & $2.365 c-f$ & $2.326 \mathrm{BCD}$ \\
\hline Chitosan 150 ppm & $2.240 \mathrm{~d}-\mathrm{g}$ & $1.953 \mathrm{hi}$ & $2.096 \mathrm{E}$ & $2.209 \mathrm{~d}-\mathrm{h}$ & $2.359 \mathrm{c}-\mathrm{f}$ & $2.284 \mathrm{BCD}$ \\
\hline Nano-chitosan 50 ppm & $2.063 \mathrm{gh}$ & 2.324 cde & 2.193 DE & $2.833 \mathrm{ab}$ & $2.773 \mathrm{ab}$ & $2.803 \mathrm{~A}$ \\
\hline Nano-chitosan 100 ppm & 2.345 cde & $2.085 \mathrm{gh}$ & 2.215 CDE & $1.597 \mathrm{k}$ & $2.546 \mathrm{bcd}$ & 2.072 DEF \\
\hline Nano-chitosan 150 ppm & 2.223 efg & $1.354 \mathrm{j}$ & $1.788 \mathrm{~F}$ & $2.647 \mathrm{bc}$ & $2.374 \mathrm{c}-\mathrm{f}$ & $2.510 \mathrm{~B}$ \\
\hline Silica $0.4 \mathrm{~g} / \mathrm{L}$ & $2.122 \mathrm{fgh}$ & $2.123 \mathrm{fgh}$ & $2.122 \mathrm{E}$ & $2.055 \mathrm{f}-\mathrm{j}$ & $1.786 \mathrm{ijk}$ & $1.921 \mathrm{EF}$ \\
\hline Silica $0.6 \mathrm{~g} / \mathrm{L}$ & $3.024 \mathrm{a}$ & $2.554 \mathrm{~b}$ & $2.789 \mathrm{~A}$ & $3.063 \mathrm{a}$ & $2.843 \mathrm{ab}$ & $2.953 \mathrm{~A}$ \\
\hline Silica $0.8 \mathrm{~g} / \mathrm{L}$ & $2.282 \mathrm{def}$ & 2.322 cde & $2.302 \mathrm{BCD}$ & $2.333 \mathrm{c}-\mathrm{f}$ & $1.946 \mathrm{~g}-\mathrm{k}$ & 2.139 CDE \\
\hline Nano-silica $0.4 \mathrm{~g} / \mathrm{L}$ & 2.183 efg & $2.414 \mathrm{bcd}$ & 2.298 BCD & $2.144 \mathrm{e}-\mathrm{i}$ & $2.286 \mathrm{c}-\mathrm{h}$ & 2.215 CD \\
\hline Nano-silica $0.6 \mathrm{~g} / \mathrm{L}$ & $2.223 \mathrm{efg}$ & $2.586 \mathrm{~b}$ & 2.404 B & $2.209 \mathrm{~d}-\mathrm{h}$ & $2.509 \mathrm{~b}-\mathrm{e}$ & $2.359 \mathrm{BC}$ \\
\hline Nano-silica $0.8 \mathrm{~g} / \mathrm{L}$ & $2.087 \mathrm{gh}$ & $2.485 \mathrm{bc}$ & 2.286 BCD & $2.364 \mathrm{c}-\mathrm{f}$ & $2.315 \mathrm{c}-\mathrm{g}$ & $2.339 \mathrm{BCD}$ \\
\hline Cultivar av. & $2.225 \mathrm{~A}$ & $2.209 \mathrm{~A}$ & & $2.290 \mathrm{~A}$ & $2.307 \mathrm{~A}$ & \\
\hline
\end{tabular}

Means having the same letter (s) within the same column are not significantly different according to Duncan's multiple range test at $5 \%$ level of probability.

Chlorophyll B and total Chlorophyll content (2.024 \& 2.071 and $3.588 \& 3.633$ $\mathrm{mg} / 100 \mathrm{mg}$ ) in the first and second seasons, respectively.

\section{Leaf mineral contents:}

Data presented in Tables 6 to 9 indicated that, there are significant varietal differences in leaf mineral content in both seasons except $\mathrm{K} \%$ in the second season and $\mathrm{P} \%$ in the first season only. Ewasy cv trees showed highest significant values of leaf mineral content compared with Zebda cv.

Effect of treatments on leaf mineral content showed significantly increase of values with nano chitosan treatments compared with other treatments. Generally, the lowest mineral contents recorded with control treatment. 
Table (6): Effect of chitosan and potassium silicate spraying on leaf nitrogen percentage of Zebda and Ewasy mango trees (2016 and 2017 seasons)

\begin{tabular}{|c|c|c|c|c|c|c|}
\hline \multirow{3}{*}{ Treatments } & \multicolumn{3}{|c|}{ First season (2016) } & \multicolumn{3}{|c|}{ Second season (2017) } \\
\hline & \multicolumn{2}{|c|}{ Cultivar } & \multirow{2}{*}{ Treat. av. } & \multicolumn{2}{|c|}{ Cultivar } & \multirow{2}{*}{ Treat. av. } \\
\hline & Zebda & Ewasy & & Zebda & Ewasy & \\
\hline Control (water) & $2.15 \mathrm{kl}$ & $2.27 \mathrm{f}-\mathrm{i}$ & 2.21 FG & $2.03 n$ & $2.19 \mathrm{k}$ & $2.11 \mathrm{H}$ \\
\hline Chitosan 50 ppm & $2.20 \mathrm{jk}$ & $2.39 \mathrm{~cd}$ & $2.29 \mathrm{DE}$ & $2.23 \mathrm{i}$ & $2.38 \mathrm{de}$ & $2.30 \mathrm{D}$ \\
\hline Chitosan 100 ppm & $2.25 \mathrm{ghi}$ & $2.21 \mathrm{jk}$ & 2.23 EFG & $2.18 \mathrm{k}$ & $2.36 \mathrm{de}$ & $2.27 \mathrm{E}$ \\
\hline Chitosan 150 ppm & $2.29 \mathrm{e}-\mathrm{i}$ & $2.42 \mathrm{bc}$ & $2.35 \mathrm{CD}$ & $2.31 \mathrm{f}$ & $2.36 \mathrm{e}$ & $2.33 \mathrm{C}$ \\
\hline Nano-chitosan 50 ppm & $2.56 \mathrm{a}$ & $2.52 \mathrm{ab}$ & $2.54 \mathrm{~A}$ & $2.46 \mathrm{~b}$ & $2.45 \mathrm{bc}$ & $2.46 \mathrm{~A}$ \\
\hline Nano-chitosan 100 ppm & $2.34 \mathrm{c}-\mathrm{g}$ & $2.39 \mathrm{~cd}$ & 2.37 BC & $2.43 \mathrm{c}$ & $2.46 \mathrm{~b}$ & $2.44 \mathrm{AB}$ \\
\hline Nano-chitosan 150 ppm & $2.35 \mathrm{c}-\mathrm{f}$ & $2.36 \mathrm{cde}$ & $2.35 \mathrm{CD}$ & $2.43 \mathrm{c}$ & $2.46 \mathrm{~b}$ & $2.45 \mathrm{~A}$ \\
\hline Silica $0.4 \mathrm{~g} / \mathrm{L}$ & $2.44 \mathrm{bc}$ & $2.43 \mathrm{bc}$ & $2.43 \mathrm{~B}$ & $2.21 \mathrm{ij}$ & 2.121 & $2.17 \mathrm{~F}$ \\
\hline Silica $0.6 \mathrm{~g} / \mathrm{L}$ & $2.20 \mathrm{jk}$ & $2.32 \mathrm{~d}-\mathrm{h}$ & $2.26 \mathrm{EF}$ & $2.03 n$ & $2.26 \mathrm{~g}$ & $2.15 \mathrm{G}$ \\
\hline Silica $0.8 \mathrm{~g} / \mathrm{L}$ & $2.22 \mathrm{ijk}$ & $2.35 c-f$ & $2.28 \mathrm{DE}$ & $2.20 \mathrm{jk}$ & $2.32 \mathrm{f}$ & $2.26 \mathrm{E}$ \\
\hline Nano-silica 0.4 g/L & 2.131 & $2.24 \mathrm{hij}$ & $2.18 \mathrm{G}$ & $2.10 \mathrm{~m}$ & $2.24 \mathrm{hi}$ & $2.17 \mathrm{~F}$ \\
\hline Nano-silica 0.6 g/L & $2.32 \mathrm{~d}-\mathrm{h}$ & $2.42 \mathrm{bc}$ & $2.37 \mathrm{BC}$ & $2.26 \mathrm{gh}$ & $2.39 \mathrm{~d}$ & $2.32 \mathrm{C}$ \\
\hline Nano-silica $0.8 \mathrm{~g} / \mathrm{L}$ & 2.24 hij & $2.42 \mathrm{bc}$ & 2.33 CD & $2.32 \mathrm{f}$ & $2.53 \mathrm{a}$ & $2.43 \mathrm{~B}$ \\
\hline Cultivar av. & $2.28 \mathrm{~B}$ & $2.37 \mathrm{~A}$ & & $2.24 \mathrm{~B}$ & $2.35 \mathrm{~A}$ & \\
\hline
\end{tabular}

Means having the same letter (s) within the same column are not significantly different according to Duncan's multiple range test at $5 \%$ level of probability. 
Table (7): Effect of chitosan and potassium silicate spraying on leaf potassium percentage of Zebda and Ewasy mango trees (2016 and 2017 seasons)

\begin{tabular}{|c|c|c|c|c|c|c|}
\hline \multirow{3}{*}{ Treatments } & \multicolumn{3}{|c|}{ First season (2016) } & \multicolumn{3}{|c|}{ Second season (2017) } \\
\hline & \multicolumn{2}{|c|}{ Cultivar } & \multirow{2}{*}{ Treat. av. } & \multicolumn{2}{|c|}{ Cultivar } & \multirow{2}{*}{ Treat. av } \\
\hline & Zebda & Ewasy & & Zebda & Ewasy & \\
\hline Control (water) & $1.67 \mathrm{k}$ & $1.72 \mathrm{j}$ & $1.69 \mathrm{~F}$ & $1.66 \mathrm{jk}$ & $1.70 \mathrm{i}$ & $1.69 \mathrm{~F}$ \\
\hline Chitosan 50 ppm & $1.56 \mathrm{p}$ & $1.61 \mathrm{o}$ & $1.58 \mathrm{I}$ & $1.55 \mathrm{~m}$ & $1.64 \mathrm{k}$ & $1.60 \mathrm{H}$ \\
\hline Chitosan 100 ppm & $1.93 \mathrm{ab}$ & $1.78 \mathrm{gh}$ & $1.86 \mathrm{~A}$ & $1.82 \mathrm{~d}$ & $1.68 \mathrm{j}$ & $1.75 \mathrm{E}$ \\
\hline Chitosan 150 ppm & $1.61 \mathrm{o}$ & $1.66 \mathrm{~lm}$ & $1.63 \mathrm{H}$ & 1.621 & $1.71 \mathrm{hi}$ & $1.67 \mathrm{G}$ \\
\hline Nano-chitosan 50 ppm & $1.63 \mathrm{~m}$ & $1.83 \mathrm{~d}$ & $1.73 \mathrm{E}$ & $1.77 \mathrm{f}$ & $1.82 \mathrm{de}$ & $1.80 \mathrm{D}$ \\
\hline Nano-chitosan 100 ppm & $1.73 \mathrm{ij}$ & $1.92 \mathrm{bc}$ & $1.82 \mathrm{~B}$ & $1.93 \mathrm{a}$ & $1.89 \mathrm{~b}$ & $1.91 \mathrm{~A}$ \\
\hline Nano-chitosan 150 ppm & $1.83 \mathrm{~d}$ & $1.77 \mathrm{~h}$ & $1.80 \mathrm{C}$ & $1.92 \mathrm{ab}$ & $1.64 \mathrm{kl}$ & $1.78 \mathrm{D}$ \\
\hline Silica $0.4 \mathrm{~g} / \mathrm{L}$ & $1.94 \mathrm{a}$ & $1.68 \mathrm{k}$ & $1.81 \mathrm{BC}$ & $1.74 \mathrm{gh}$ & $1.77 \mathrm{f}$ & $1.75 \mathrm{E}$ \\
\hline Silica $0.6 \mathrm{~g} / \mathrm{L}$ & $1.64 \mathrm{~m}$ & $1.72 \mathrm{j}$ & $1.68 \mathrm{G}$ & $1.74 \mathrm{gh}$ & $1.64 \mathrm{k}$ & $1.69 \mathrm{~F}$ \\
\hline Silica $0.8 \mathrm{~g} / \mathrm{L}$ & $1.60 \mathrm{o}$ & $1.74 \mathrm{i}$ & $1.67 \mathrm{G}$ & $1.65 \mathrm{k}$ & $1.86 \mathrm{c}$ & $1.75 \mathrm{E}$ \\
\hline Nano-silica $0.4 \mathrm{~g} / \mathrm{L}$ & $1.75 \mathrm{i}$ & $1.82 \mathrm{de}$ & $1.79 \mathrm{D}$ & $1.80 \mathrm{e}$ & $1.85 \mathrm{c}$ & $1.83 \mathrm{C}$ \\
\hline Nano-silica $0.6 \mathrm{~g} / \mathrm{L}$ & $1.80 \mathrm{fg}$ & $1.81 \mathrm{def}$ & $1.81 \mathrm{C}$ & $1.75 \mathrm{fg}$ & $1.76 \mathrm{fg}$ & $1.75 \mathrm{E}$ \\
\hline Nano-silica $0.8 \mathrm{~g} / \mathrm{L}$ & $1.81 \mathrm{ef}$ & $1.90 \mathrm{c}$ & $1.85 \mathrm{~A}$ & $1.81 \mathrm{de}$ & $1.92 \mathrm{a}$ & $1.87 \mathrm{~B}$ \\
\hline Cultivar av. & $1.73 \mathrm{~B}$ & $1.77 \mathrm{~A}$ & & $1.75 \mathrm{~A}$ & $1.76 \mathrm{~A}$ & \\
\hline
\end{tabular}

Means having the same letter (s) within the same column are not significantly different according to Duncan's multiple range test at $5 \%$ level of probability. 
Table (8): Effect of chitosan and potassium silicate spraying on leaf phosphorus percentage of Zebda and Ewasy mango trees (2016 and 2017 seasons)

\begin{tabular}{|c|c|c|c|c|c|c|}
\hline \multirow{3}{*}{ Treatments } & \multicolumn{3}{|c|}{ First season (2016) } & \multicolumn{3}{|c|}{ Second season (2017) } \\
\hline & \multicolumn{2}{|c|}{ Cultivar } & \multirow{2}{*}{ Treat. av. } & \multicolumn{2}{|c|}{ Cultivar } & \multirow{2}{*}{ Treat. av. } \\
\hline & Zebda & Ewasy & & Zebda & Ewasy & \\
\hline Control (water) & $0.205 \mathrm{hi}$ & $0.235 \mathrm{c}-\mathrm{i}$ & $0.220 \mathrm{CD}$ & $0.193 \mathrm{q}$ & $0.225 \mathrm{o}$ & $0.209 \mathrm{~K}$ \\
\hline Chitosan 50 ppm & $0.237 \mathrm{~b}-\mathrm{i}$ & $0.274 \mathrm{a}-\mathrm{d}$ & $0.256 \mathrm{ABC}$ & $0.252 \mathrm{k}$ & $0.293 \mathrm{~b}$ & 0.273 D \\
\hline Chitosan 100 ppm & $0.222 \mathrm{e}-\mathrm{i}$ & $0.235 \mathrm{c}-\mathrm{i}$ & 0.229 CDE & $0.203 \mathrm{p}$ & $0.225 \mathrm{o}$ & $0.214 \mathrm{~J}$ \\
\hline Chitosan 150 ppm & $0.244 \mathrm{~b}-\mathrm{i}$ & $0.264 \mathrm{a}-\mathrm{f}$ & $0.254 \mathrm{ABC}$ & $0.266 \mathrm{~h}$ & $0.282 \mathrm{e}$ & $0.274 \mathrm{D}$ \\
\hline Nano-chitosan 50 ppm & 0.266 a-e & $0.282 \mathrm{abc}$ & $0.274 \mathrm{~A}$ & $0.273 \mathrm{~g}$ & $0.302 \mathrm{a}$ & $0.288 \mathrm{~A}$ \\
\hline Nano-chitosan 100 ppm & $0.274 \mathrm{a}-\mathrm{d}$ & $0.253 \mathrm{a}-\mathrm{h}$ & $0.264 \mathrm{AB}$ & $0.263 \mathrm{i}$ & $0.236 \mathrm{mn}$ & $0.249 \mathrm{H}$ \\
\hline Nano-chitosan 150 ppm & $0.278 \mathrm{abc}$ & $0.285 \mathrm{ab}$ & $0.281 \mathrm{~A}$ & $0.263 \mathrm{i}$ & $0.305 \mathrm{a}$ & 0.284 B \\
\hline Silica $0.4 \mathrm{~g} / \mathrm{L}$ & 0.266 a-e & $0.235 \mathrm{c}-\mathrm{i}$ & 0.251 A-D & $0.252 \mathrm{k}$ & $0.252 \mathrm{jk}$ & $0.252 \mathrm{G}$ \\
\hline Silica $0.6 \mathrm{~g} / \mathrm{L}$ & 0.209 ghi & $0.246 \mathrm{~b}-\mathrm{h}$ & 0.228 CDE & $0.232 \mathrm{n}$ & $0.278 \mathrm{f}$ & $0.255 \mathrm{~F}$ \\
\hline Silica $0.8 \mathrm{~g} / \mathrm{L}$ & $0.213 \mathrm{f}-\mathrm{i}$ & $0.260 \mathrm{a}-\mathrm{g}$ & 0.236 B-E & $0.236 \mathrm{~m}$ & 0.2421 & 0.239 I \\
\hline Nano-silica $0.4 \mathrm{~g} / \mathrm{L}$ & $0.214 \mathrm{f}-\mathrm{i}$ & $0.254 \mathrm{a}-\mathrm{h}$ & 0.234 B-E & 0.2391 & $0.272 \mathrm{~g}$ & $0.256 \mathrm{~F}$ \\
\hline Nano-silica 0.6 g/L & $0.270 \mathrm{a}-\mathrm{d}$ & $0.296 \mathrm{a}$ & $0.283 \mathrm{~A}$ & $0.264 \mathrm{i}$ & $0.290 \mathrm{c}$ & $0.277 \mathrm{C}$ \\
\hline Nano-silica $0.8 \mathrm{~g} / \mathrm{L}$ & $0.223 \mathrm{~d}-\mathrm{i}$ & $0.197 \mathrm{i}$ & $0.210 \mathrm{E}$ & $0.255 \mathrm{j}$ & $0.286 \mathrm{~d}$ & $0.270 \mathrm{E}$ \\
\hline Cultivar av. & $0.240 \mathrm{~A}$ & $0.255 \mathrm{~A}$ & & 0.245 B & $0.268 \mathrm{~A}$ & \\
\hline
\end{tabular}

Means having the same letter (s) within the same column are not significantly different according to Duncan's multiple range test at $5 \%$ level of probability. 
Table (9): Effect of chitosan and potassium silicate spraying on leaf zinc content (ppm) of Zebda and Ewasy mango trees (2016 and 2017 seasons)

\begin{tabular}{|c|c|c|c|c|c|c|}
\hline \multirow{3}{*}{ Treatments } & \multicolumn{3}{|c|}{ First season (2016) } & \multicolumn{3}{|c|}{ Second season (2017) } \\
\hline & \multicolumn{2}{|c|}{ Cultivar } & \multirow{2}{*}{ Treat. av. } & \multicolumn{2}{|c|}{ Cultivar } & \multirow{2}{*}{ Treat. av. } \\
\hline & Zebda & Ewasy & & Zebda & Ewasy & \\
\hline Control (water) & $28.12 \mathrm{q}$ & $29.13 n$ & $28.63 \mathrm{~K}$ & $27.48 \mathrm{u}$ & $28.64 \mathrm{q}$ & $28.06 \mathrm{~L}$ \\
\hline Chitosan 50 ppm & $31.46 \mathrm{~g}$ & $28.12 \mathrm{q}$ & 29.79 I & $31.52 \mathrm{~g}$ & $30.22 \mathrm{~m}$ & $30.87 \mathrm{~F}$ \\
\hline Chitosan 100 ppm & $30.15 \mathrm{~m}$ & $28.34 \mathrm{p}$ & 29.24 J & 29.70 o & $30.60 \mathrm{k}$ & 30.15 G \\
\hline Chitosan 150 ppm & $31.80 \mathrm{f}$ & $30.13 \mathrm{~m}$ & $30.97 \mathrm{~F}$ & $31.82 \mathrm{e}$ & $28.17 \mathrm{~s}$ & 29.99 H \\
\hline Nano-chitosan 50 ppm & $28.32 \mathrm{p}$ & $33.23 \mathrm{~b}$ & $30.78 \mathrm{G}$ & $30.17 \mathrm{n}$ & $33.35 \mathrm{~b}$ & $31.76 \mathrm{~B}$ \\
\hline Nano-chitosan 100 ppm & $31.22 \mathrm{~h}$ & $31.06 \mathrm{i}$ & $31.14 \mathrm{E}$ & $28.83 \mathrm{p}$ & $31.12 \mathrm{~h}$ & $29.98 \mathrm{H}$ \\
\hline Nano-chitosan 150 ppm & $31.82 \mathrm{f}$ & $33.40 \mathrm{a}$ & $32.61 \mathrm{~A}$ & 30.441 & $33.64 \mathrm{a}$ & 32.04 A \\
\hline Silica $0.4 \mathrm{~g} / \mathrm{L}$ & $32.13 \mathrm{e}$ & $30.72 \mathrm{j}$ & 31.42 D & 30.461 & $28.12 \mathrm{t}$ & 29.29 I \\
\hline Silica $0.6 \mathrm{~g} / \mathrm{L}$ & $28.61 \mathrm{o}$ & $28.62 \mathrm{o}$ & $28.62 \mathrm{~K}$ & $26.34 \mathrm{v}$ & $30.20 \mathrm{mn}$ & $28.27 \mathrm{~K}$ \\
\hline Silica $0.8 \mathrm{~g} / \mathrm{L}$ & $30.12 \mathrm{~m}$ & 30.331 & $30.22 \mathrm{H}$ & $28.53 \mathrm{r}$ & $28.55 \mathrm{r}$ & 28.54 J \\
\hline Nano-silica $0.4 \mathrm{~g} / \mathrm{L}$ & $30.16 \mathrm{~m}$ & $32.15 \mathrm{e}$ & $31.16 \mathrm{E}$ & $30.21 \mathrm{~m}$ & $31.67 \mathrm{f}$ & $30.94 \mathrm{E}$ \\
\hline Nano-silica $0.6 \mathrm{~g} / \mathrm{L}$ & $31.05 \mathrm{i}$ & $32.62 \mathrm{~d}$ & 31.84 B & $30.64 \mathrm{j}$ & $32.09 \mathrm{~d}$ & 31.37 D \\
\hline Nano-silica $0.8 \mathrm{~g} / \mathrm{L}$ & $30.46 \mathrm{k}$ & $33.04 \mathrm{c}$ & $31.75 \mathrm{C}$ & $30.78 \mathrm{i}$ & $32.16 \mathrm{c}$ & $31.47 \mathrm{C}$ \\
\hline Cultivar av. & $30.42 \mathrm{~B}$ & $30.84 \mathrm{~A}$ & & $29.76 \mathrm{~B}$ & $30.66 \mathrm{~A}$ & \\
\hline
\end{tabular}

Means having the same letter (s) within the same column are not significantly different according to Duncan's multiple range test at $5 \%$ level of probability.

The interaction between cultivars $\times$ treatments recorded significantly the highest values of leaf nitrogen percentage from Zebda $\mathrm{cv} \times 50 \mathrm{ppm}$ nano chitosan in the first season and $0.8 \mathrm{~g} / \mathrm{l}$ nano silica in the second season. While, 
the lowest values came from, generally, Zebda $\mathrm{cv} \times$ control during both seasons. The values of leaf potassium percentage was significantly the highest in Zebda $\mathrm{cv} \times 0.4 \mathrm{~g} / \mathrm{l}$ silica in the first season and $100 \mathrm{ppm}$ nano chitosan in the second season. But, the lowest values came from Zebda $\mathrm{cv} \times 0.6 \mathrm{~g} / \mathrm{l}$ silica in the first season and $50 \mathrm{ppm}$ chitosan in the second season. The highest values of leaf phosphorus percentage, obtained from Ewasy $\mathrm{cv} \times$ nano chitosan treatments during the two seasons. The lowest values came from Ewasy $\mathrm{cv} \times$ nano silica 0.8 $\mathrm{g} / \mathrm{l}$ treatment in the first season and $0.6 \mathrm{~g} / \mathrm{l}$ silica with Zebda cv in the second season. The values of leaf zinc content (ppm) was significantly the highest in Ewasy cv and 150 ppm nano chitosan treatment in the first and second seasons. While, the lowest values came from Ewasy cv and control treatment in the first season and $50 \mathrm{ppm}$ nano chitosan with Zebda cv in the second season.

\section{DISCUSSION}

Ewasy cv. displayed the higher effect in most cases of their tested attributes (average leaf area leaf, content of Chlorophyll B \& total and mineral) compared with Zebda cv. Differences between the two mango cvs in all parameters are due to varietal differences that go back to genetic composition. In this respect, Bally (2006) reported that growth vigor of a mango cv. is an inherent property ascribing to the genetic make-up of the cultivar. Outweigh of a mango cv in growth traits especially the area of photosynthetic leaves indicates its higher capacity for accumulating photosynthesis. It is well known that mango cvs; as any other plant cultivars; differ greatly in response of their genetic make up to the environmental factors that affecting developmental processes and ability to thrive benefit from the available growth factors. (Zuo et al., 2007).

Results indicated that, tested treatments were improving leaf content of pigments and minerals especially silica at $0.6 \mathrm{~g} / \mathrm{L}$ and nano chitosan $50 \mathrm{ppm}$ treatments. The obtained results are in agreement with those Hussein and Radwan (2017) on mango, Mondal et al. (2012) on okra, Mondal et al. (2013) on mungbean and Esraa, (2017) on prime seedless grapevines by treatment of chitosan improved growth aspects and leaf content of pigment and mineral. Silicon were favorable in enhancing growth aspects, leaf pigments and nutrients content (Gad El-Kareem et al., 2014, Omar, 2015, Ahmed et al., 2017, Youssef, 2017 and Faissal et al., 2018). 
Zong et al. (2016) in edible rape (Brassica rapa L.) found that, foliar application of chitosan promoted the plant growth and leaf chlorophyll contents. So, Pereira et al. (2017) reported that, bioactivity assays using Phaseolus vulgaris showed that the alginate/chitosan (ALG/CS)-GA3 nanoparticles were most effective in increasing leaf area and the levels of chlorophylls and carotenoids.

Silicon has many functions in plant nutrition. It has many regulatory roles in enhancing the tolerance of plants to biotic and abiotic stresses, water retention, photosynthesis, plant pigments and building of carbohydrates and natural growth regulators (Gang et al., 2003, Lux et al., 2003, Ma, 2004 and Hattori et al., 2005).

Conclusively, from results of this research, the resembling conditions that spraying mango trees nano- nano chitosan 50 and $150 \mathrm{ppm}$ nano-silica 0.6 and $0.8 \mathrm{~g} / \mathrm{L}$ before flowering is desirable for improving leaf characteristics it is recommending, under Belbeis district, Sharkia Governorate, Egypt

\section{REFERENCES}

Ahmed, F.F. and M.H. Morsy (1999). A new method for measuring leaf area in different fruit species. J. of Agric. Rec. \& Dev. 19: 97-105.

Ahmed, M.M.A., F. A. Faissal., I.M. I. Hamdy., S.A. S. Abbas, and N. R. S. Magdy (2017). Effect of spraying silicon and some nutrients on growth, palm nutritional status, yield and fruit quality of zaghloul date palms. 1Effect of spraying silicon and some nutrients on growth and palm nutritional status of zaghloul date palms. J. Product. \& Dev., 22(3): 585 -600 .

Al-Hetar, M. Y., M. A. Zainal Abidin., M. Sariah and M.Y. Wong (2011). Antifungal activity of chitosan against Fusarium oxysporum f. sp. cubense. J. Appl. Polym. Sci., 120(4): 2434-2439.

Bally, I.S.E. (2006). Species profiles of Pacific Island Agrofrosty. Perm. Agri. Res., 1- 25.

Bittelli, M., M. Flury., G.S. Campbell, and E.J. Nichols, (2001). Reduction of transpiration through foliar application of chitosan. Agric. and Forest Meteorology, 107(3):167-175. 
Chapman, H. D. and P. F. Pratt, (1961). Methods of Analysis for soil, Plant and Waters. University of California, Division of Agriculture Science.

Chibu, H. and H. Shibayama, (1999). Effects of chitosan applications on the early growth of several crops. Report of Kyushu Branch of the Crop Sci. Soc. of Japan, 65: 83-87.

Devlieghere, F., A.Vermeulen and J. Debevere, (2004). Chitosan: antimicrobial activity, interactions with food components and applicability as a coating on fruit and vegetables. Food microbiology, 21(6): 703-714.

Dias, A. M. A., A. R. Cortez., M. M. Barsan., J. B. Santos., C. M. A. Brett, and H. C. De Sousa, (2013). Development of greener multi-responsive chitosan biomaterials doped with biocompatible ammonium ionic liquids. ACS Sustainable Chem. Eng., 1(11): 1480-1492.

Du, J., H.Gemma., S. Iwahori, 1997. Effects of chitosan coating on the storage of peach, Japanese pear and kiwifruit. J. Jpn. Soc. Hort. Sci. 66, 15_22.

Duncan, D.B. (1955). Multiple range and Multiple F test. Biometrics, 11:1- 42.

Dzung, N. A., V. T. P. Khanh and T. T. Dzung (2011). Research on impact of chitosan oligomers on biophysical characteristics, growth, development and drought resistance of coffee. Carbohydr. Polym., 84(2): 751-755.

Faissal F. A., H.M. A. Ahmed ., M.A. Salah- Eldeen and A. M. Mohamed (2018). Trials for improving the productivity and reducing shot berries in superior grapevines by using silicon and glutathione. J. Product. \& Dev., 23(1): 25-39.

Gad El- Kareem. M.R., A.M.K. Abdelaal and A.Y. Mohamed (2014). the synergistic effect of using silicon and selenium on fruiting of Zaghloul date palm (Phoenic dectylifera L.) World Academy of ci. Engineering and Technology, Inter. J. of Agric. Biosystems Sci. and Engineering, 8 (3):959-964.

Gang, H.J.K., K.M. Chen., G.C. Chen., S.M. Wan and C.L. Zhang (2003). Effect of silicon on growth of wheat under drought. H. Plant. Nutr. , 26(5):1055-1063.

Hattori, T., S. Inanaga., H. Araki., P. An., S. Mortia., M. Luxova and A. Lux (2005). Application of silicon enhanced drought tolerance in sorghum bicolor. Physiolgia Plantarum. 123: 459 - 466. 
Hussein. Esraa. M. E. 2017. Behviour of prime seedless grapevines grown under sandy soil conditions to foliar application of chitosan. J. Product. \& Dev., 22(3): 683-696.

Hussein S. H.M and E.M.A. Radwa (2017). Insight into the effect of chitosan on growth and fruiting of succary mango trees. J. Product. \& Dev., 22(3): 781- 793 .

Jabeen, N. and R. Ahmad (2013). The activity of antioxidant enzymes in response to salt stress in safflower (Carthamus tinctorius L.) and sunflower (Helianthus annuus L.) seedlings raised from seed treated with chitosan. J. Sci. Food Agric., 93(7):1699-1705.

Li, H. and T. Yu (2000). Effect of chitosan on incidence of brown rot, quality and physiological attributes of postharvest peach fruit. Journal of the Science of Food and Agric., 81(2): 269-274.

Lizárraga-Paulín, E. G., I.Torres-Pacheco., E. Moreno-Martínez and S. P. Miranda-Castro (2011). Chitosan application in maize (Zea mays) to counteract the effects of abiotic stress at seedling level. Afr. J. Biotechnol., 10(3): 6439-6446.

Lux, A., M. Luxova., J. Abe., E. Tanmoto and S. Inanaga (2003). The dynamic of silicon deposition in the sorghum root endodermis. New Physiol., 158:437-441.

Ma, J.F. (2004). Role of silicon in enhancing the resistance of plants to biotic and abiotic stresses. Soil Scr. Plant Nutr. 50:11-18.

Mondal M.M.A., M.A. Malek., A.B. Puteh. M.R. Ismail., M. Ashrafuzzaman and L. Naher (2012). Effect of foliar application of chitosan on growth and yield in okra. Aust. J. Crop Sci. 6(5):918-921.

Mondal, M.M.A., M.A. Malek., A.B. Puteh and M.R. Ismail (2013). Foliar application of chitosan on growth and yield attributes of mungbean (Vigna radiata L.) Wilczek. Bangladesh J. Bot., 42(1): 179-183.

Mukherjee, S.K. (1951). Origin of mango. Indian J. Genet. Pl. Breed. 11: 49-55.

Omar, A.I.A. (2015). Effect of spraying seaweed extract and potassium silicate on growth and fruiting of Al- Saidey date palms. M. Sc. Thesis Fac. of Agric. Minia Univ. Egypt.

Pereira A. E. S., P. M. Silva., J. L. Oliveira., H. C. Oliveira and L. F. Fraceto (2017). Chitosan nanoparticles as carrier systems for the plant growth hormone gibberellic acid. Colloids and Surfaces B: Bio interfaces, 150: 141-152. 
Pongprayoon, W., S.Roytrakul., R. Pichayangkura and S. Chadchawan (2013). The role of hydrogen peroxide in chitosan-induced resistance to osmotic stress in rice (Oryza sativa L.). Plant Growth Regul., 70(2):159173.

Snedecor. G. W. and W.G. Cochran (1980). Statistical Methods. Sixth Edition Ioa State Unit. Press, Ames, Iowa, U. S. pp 1207 - 1285.

Wettestein, D.V. (1957). Chlorophyll, lethal und der Submikroscopische Formwechsel der plastiden. Expt . Cell-Research, 12: 427-433.

Yousef, M.S.M. (2017). Effect of spraying silicon on fruiting of Sakkoti date palms. M.Sc. Thesis Fac. of Agric. Minia Univ. Egypt.

Zong H., S. Liu., R. Xing., X. Chen and P. Li (2016). Protective effect of chitosan on photosynthesis and antioxidative defense system in edible rape (Brassica rapa L.) in the presence of cadmium. Carbohydrate Polymers. 154: 241 - 246.

Zuo Y., L. Ren., F. Zhang and R.F. Jiang (2007). Bicarbonate concentration as affected by soil water content controls iron nutrition of peanut plants in a calcareous soil. Plant Phys. and Bio., 45: 357-364.

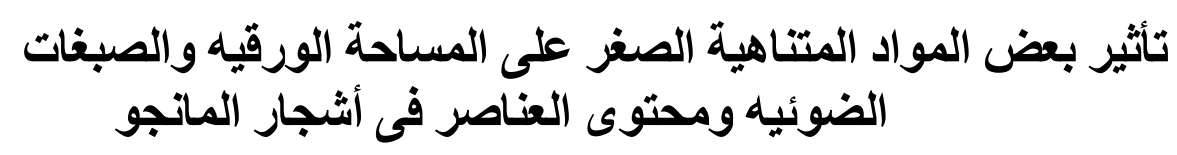

طارق غريب عطية عليوة' ، أسامه أحمد أبراهيم زقزوق ' ، محمد ممتاز جاد' ،

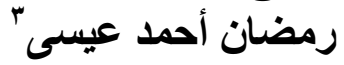

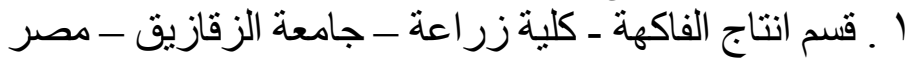

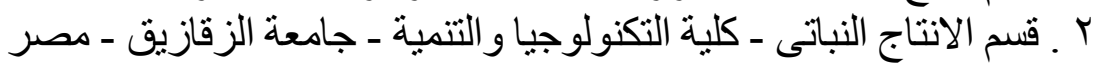

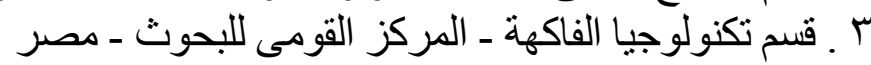

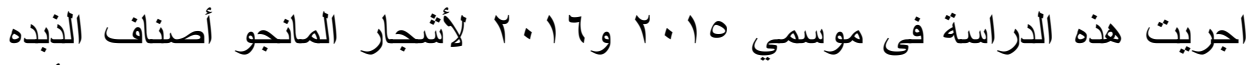

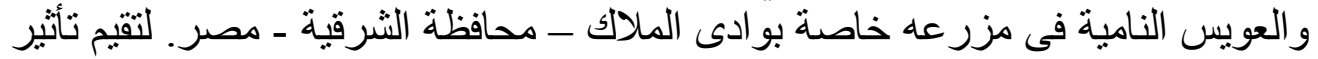

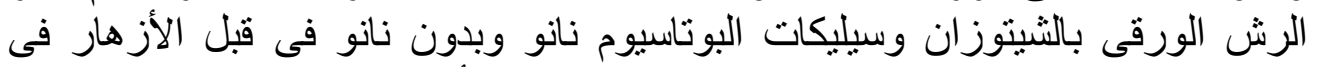
منتصف فبر اير على متوسط المساحة الورقية ومحتوى الأوراق من الصبنات فئن ونات العناصر. 
تفوق صنف العويس بالمقارنة بالصنف الذبده فى لمعظم الصفات المختبرة - كلورفيا أ ، ب و الكلى و أيضا العناصر المعدنية.

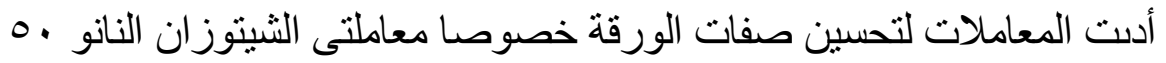

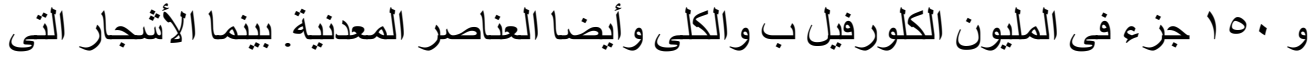

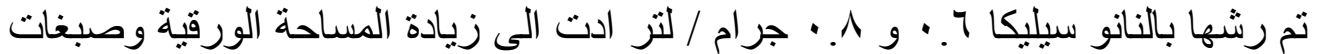

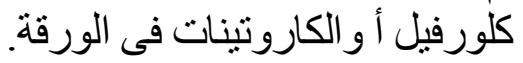
التوصية: من نتائج هذه الدراسة نتصح تحت ظروف فات في منطقة صحر اء بلبيس

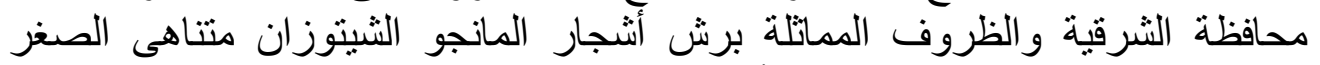

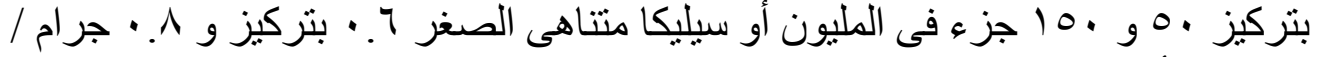

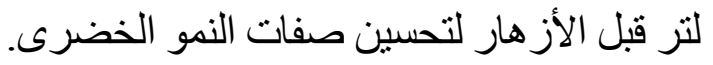

